\section{In the beginning}

Gerald F. Joyce

Information and the Origin of Life. By

Bernd-Olaf Küppers. MIT Press: 1990.

Pp. 215. £20.25, \$22.25.

MOLECULAR biology has been remarkably successful in following a reductionist programme aimed at providing a detailed understanding of biological phenomena in terms of the laws of physics and chemistry. It is natural to wonder to what degree this programme can be extended toward understanding the origin of life itself. All of biology rests on the foundation of neodarwinism, drawing on the principles of population biology and molecular genetics. A 'molecular-darwinistic' approach, developed by Manfred Eigen and others over the past 20 years, has been used successfully to extend neodarwinism to the level of a population of informational macromolecules. In his scholarly and intensely thought-provoking book, Küppers presents the case for molecular darwinism as he examines the philosophical underpinnings of the problem of the origin of biological information.

Küppers considers three possible explanations for the origin of biological information. The chance hypothesis, which he attributes to Jacques Monod, suggests that the specific sequence of the first genetic molecule was the result of a purely random process on the primitive Earth. Thus the origin of life was a unique and entirely unpredictable event. Küppers rejects this hypothesis on statistical grounds and, furthermore, applies algorithmic information theory to show that the chance hypothesis is inherently unprovable. The teleological approach, attributable to Michael Polanyi and others, argues that living systems possess a higher regulatory principle that goes beyond the laws of physics and chemistry and allows order to emerge against the universal tendency toward maximum entropy. Küppers rejects this hypothesis as well, pointing to the results of nonequilibrium thermodynamics which explain how order can emerge in an open system not at equilibrium. Again he turns to algorithmic information theory, this time to show that the teleological approach is inherently unfalsifiable.

Between the chance hypothesis and the teleological approach lies the moleculardarwinistic approach. According to this model, the origin of biological information results from the interplay between purely random mutational events and the higher-order principle of natural selection. Küppers firmly rejects any notion that natural selection is a special property of living systems, noting that while "natural selection in itself is not a physical law neither is it an irreducible property of living systems ... rather, it results as a direct consequence of physical laws" (page 147). He describes in precise terms, following the work of Eigen, how selection can be characterized by an optimization principle that is a direct consequence of the behaviour of a population of selfreplicating macromolecules that have different rates of production. Natural selection, therefore, does not succumb to the tautology 'survival of the survivor', but rather is a reflection of the quantifiable behaviour of self-replicating entities.

Küppers' refutation of the oftenrepeated statement that biological organisms can be understood only through a holistic approach that recognizes that "the whole is greater than the sum of the parts" is a treat for those who reject such fuzzy-headed thinking. Although he acknowledges that the methodology of the holistic approach often has heuristic value, he cautions against allowing "methodological mysticism to creep back into biology, at a time when this had only just begun to be eliminated by the development of molecular biology" (page 175).

$A$ reductionist to the core, Küppers nonetheless has trouble establishing criteria to distinguish the living from the nonliving. Metabolism, self-reproduction and mutability are obviously necessary attributes, but are they sufficient to exclude inanimate structures such as crystals and viruses? Küppers adopts the uncomfortable position that "the transition from nonliving to living is a continuous one" (page 133). To him, a popula- tion of self-replicating nucleic acids undergoing darwinian evolution lies in the realm of the prebiotic. What is lacking, in his view, is protein-based catalytic function. Others might argue that metabolism, self-reproduction and (heritable) mutability are sufficient criteria for life, thereby equating the origin of darwinian evolution, whatever its chemical basis, with the origin of life. But then the moleculardarwinistic approach would address the early evolution of life rather than the origin of life and one would be left without an explanation for the appearance of the first genetic molecule.

The only serious shortcoming of this book is that, despite a 1990 copyright, it is somewhat out of date. There are virtually no references to the scientific literature of the past 10 years, including no mention of catalytic RNA and no discussion of the recent work by Eigen on the movement of an evolving population through sequence space. The book was first published in German in 1986 and has apparently not been revised before publication of the English translation. Nonetheless, this is an important contribution to the philosophy of science. It merits serious consideration by biologists and by those interested in the question of how biology arose when once there was only physics and chemistry.

Gerald $F$. Joyce is in the departments of Chemistry and of Molecular Biology, Research Institute of Scripps Clinic, La Jolla, California 92037, USA.

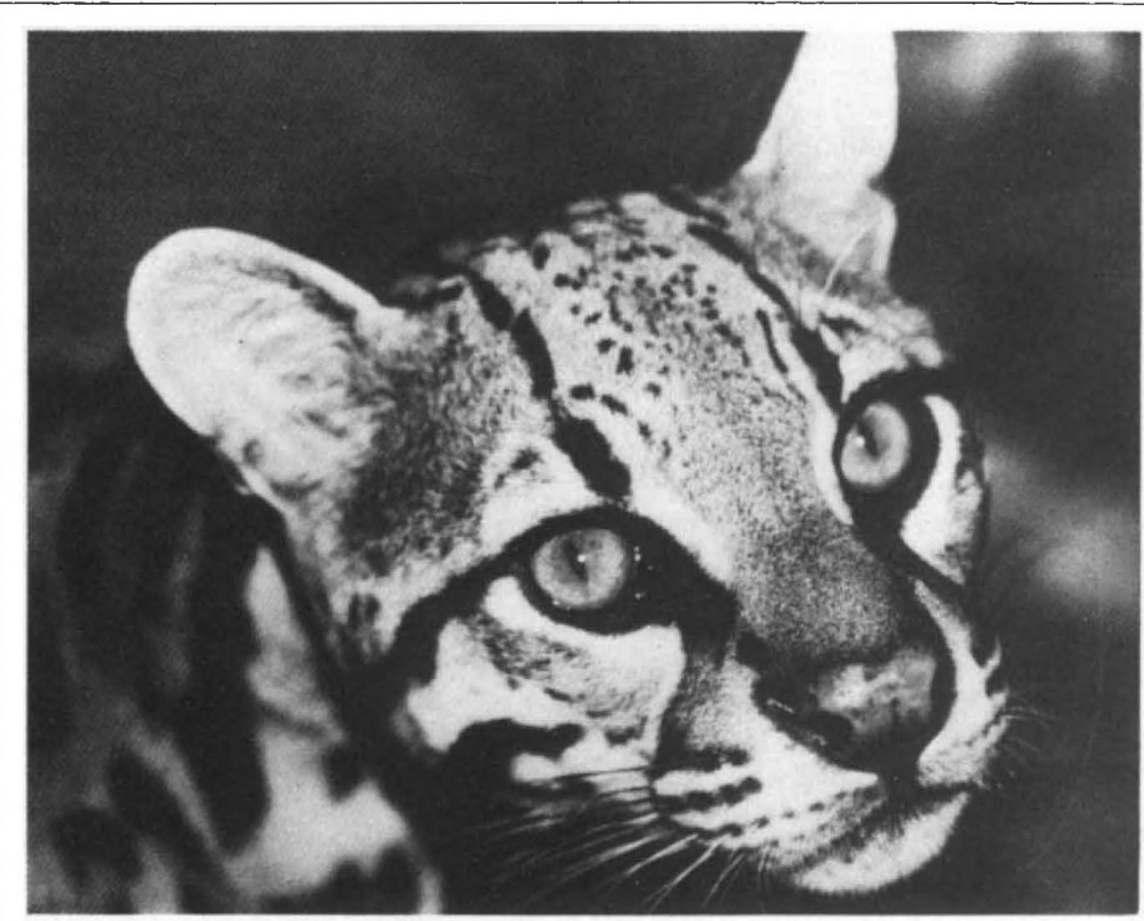

This ocelot, Felis paradalis, is one of 36 species of wild cats in an "action plan for species conservation". From Conserving the World's Biological Diversity by J. A. McNeely et al., published by the World Bank. The authors explain the importance of diversity and call for action from businesses and institutions to preserve biological resources. Price is $\$ 14.95$ (pbk). (Photo by A. Young.) 